This work is licensed under a Creative Commons Attribution 4.0 International License
https://creativecommons.org/licenses/by/4.0/
RESEARCH ARTICLE I HAY4HAЯ CTATb9
CUstinova L.P., Filina I.A., Varenyk G.V., Maltsev E.A., 2021
Принята 20.05 .2021 | Accepted 20.05.2021

\title{
ANALYSIS OF POPULATION SATISFACTION WITH THE QUALITY OF PROVIDING SERVICES ON PREMIUM MEDICINAL PROVISION
}

\author{
Ustinova $^{1}$ L.P., Filina ${ }^{1}$ I.A., Varenyk ${ }^{2}$ G.V., Maltsev ${ }^{1}$ E.A. \\ ${ }^{I}$ Oryol State University named after I.S. Turgenev, Oryol, Russian Federation \\ ${ }^{2}$ Belgorod State National Research University, Belgorod, Russian Federation
}

\section{АНАЛИЗ УДОВЛЕТВОРЕННОСТИ НАСЕЛЕНИЯ КАЧЕСТВОМ ПРЕДОСТАВЛЕНИЯ УСЛУГ ПО ЛЬГОТНОМУ ЛЕКАРСТВЕННОМУ ОБЕСПЕЧЕНИЮ}

\author{
Устинова $^{1}$ Л.П., Филина ${ }^{1}$ И.А., Вареных ${ }^{2}$ Г.В., Мальцев ${ }^{1}$ Е.А. \\ ${ }^{1}$ Орловский государственный университет имени И.С. Тургенева, г. Орёл, Российская Федерация \\ ${ }^{2}$ Белгородский государственный национальный исследовательский университет, \\ г. Белгород, Российская Федерачии
}

Abstract. Drug therapy is one of the main components of medical care for the population, and the provision of drugs is an important indicator of its availability and quality. The purpose of our work was to analyze satisfaction with the quality of the provision of services for preferential drug provision. The works of domestic and foreign scientists in the field of preferential drug provision, as well as regulatory and organizational documentation on pharmaceutical activities were taken as an information base. The survey method served as the main research method. The objects of the study were the visitors of the pharmacy organizations of the Oryol region, who receive drugs under the program of preferential drug provision. The study found that $49.0 \%$ of respondents had difficulties in making an appointment with a doctor; cases of refusal to issue a prescription were indicated by $29.0 \%$ of the respondents; $45.0 \%$ of the respondents had to spend a long time on obtaining a preferential prescription for a drug by the decision of a medical commission. The majority of respondents $(54.0 \%)$ do not receive medicines on preferential terms on time (postponed for more than 10 days). Almost half of the surveyed respondents (48.0\%) are
Аннотация. Лекарственная терапия является одним из главных составляюших медицинской помощии населения, а обеспечение лекарствами служит важным показателем ее доступности и качества. Целью нашей работь явился анализ удовлетворенности качеством предоставления услуг по льготному лекарственному обеспечению. $B$ качестве информационной базы были взяты труды отечественных и зарубежных ученых в области льготного лекарственного обеспечения, а также нормативно-правовая $u$ организационная документачия по фармачевтической деятельности. Метод анкетирования послужил основным методом исследования. Объектами исследования стали посетители аптечных организаций Орловской области, получающие лекарственные препараты в рамках программы льготного лекарственного обеспечения. В результате исследования выяснилось, что имели трудности при записи на прием к врачу 49,0\% респондентов; на случаи отказа в выписке льготного рецепта указали 29,0\% опрошенных ; $45,0 \%$ анкетируемым приходилось затрачивать

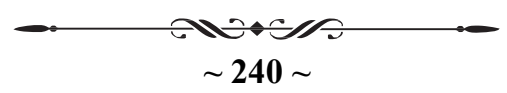


not satisfied with the treatment with prescription drugs prescribed under the program of preferential drug provision, due to the lack of drugs prescribed by a doctor in the pharmacy organization at the time of contact and their preference for drugs from other manufacturers. Most of the respondents $(61.0 \%)$ consider the range of preferential drugs to be insufficient for the treatment of their underlying disease. Some respondents (20.0\%) are not satisfied with the service in a pharmacy organization; $19.0 \%$ are dissatisfied with the quality and completeness of information about medicines received from a doctor and a pharmacy worker. The results obtained were communicated to the management of medical and pharmaceutical organizations serving privileged categories of citizens in the Oryol region. The authors of the study developed a Standard Operating Procedure "Procedure for Selling Medicines to Beneficial Categories of Citizens", which helps to optimize the quality of service in pharmacies for the population receiving medicines under the preferential drug provision program.

Keywords: pharmaceutical provision, medicines, privileged categories of citizens, medical assistance. продолжительное время на оформление льготного рецепта на лекарственный препарат решением врачебной комиссии. Большинство респондентов (54,0\%) не получают своевременно лекарственные препараты по льготным рецептам (отложено на срок более 10 дней). Почти половина опрошенных респондентов $(48,0 \%)$ не удовлетворены лечением лекарственными препаратами по рецептам, выписанным в рамках программы льготного лекарственного обеспечения, по причине отсутствия выписанных врачом лекарственных препаратов в аптечной организачии на момент обращения $u$ предпочтения ими лекарственных препаратов иных фирм-производителей. Большая часть анкетируемых (61,0\%) считает ассортимент льготных лекарственных препаратов недостаточным для лечения своего основного заболевания. Некоторых респондентов (20,0\%) не устраивает обслуживание в аптечной организации; 19,0\% недовольны качеством u полнотой информации о лекарственных препаратах, полученной от врача и аптечного работника. Полученные результаты были доведены до руководства медицинских $u$ аптечных организаций, обслуживающих льготные категории граждан в Орловской области. Авторами исследования разработана Стандартная операчионная прочедура "Порядок реализации лекарственных препаратов льготным категориям граждан», которая способствует оптимизаиии качества обслуживания в аптеках населения, получающего лекарственные препараты в рамках программы льготного лекарственного обеспечения.

Ключевые слова: лекарственное обеспечение, лекарственные препараты, льготные категории граждан, медицинская помощь.

\section{REFERENCES}

[1] Gracheva N.N., Zinovieva A.V., Polyakova E.V. Topical issues of preferential provision of citizens under state programs // Materials of the VIII international scientific and practical conference "Solving the problems of enterprise development: the role of scientific research." 2016. P. 1 - 9.

[2] Kovaleva K.A., Narkevich I.A., Nemyatykh O.D., Vasyagina Yu.A. Analysis of federal programs of preferential drug provision of the population // Remedium. 2019. Vol. 3. P.54-58.

[3] Feoktistova Yu. V., Poddubny E. A., Maneyeva E. S., Eliseeva E. V. Regulatory and organizational foundations of preferential drug provision for certain categories of citizens // Tavrichesky medicobiological bulletin. 2018, Vol. 21, No. 4, P. 120-127.

[4] Khabriev R.U., Sukhodolov A.P., Spasennikov B.A., Bezmelnitsyna L.Yu., Meshkov D.O. Decisionmaking system in the program of preferential drug provision // Bulletin of the Baikal State University. 2018. Vol.28, No. 1. P. 6-11.

\section{БИБЛИОГРАФИЧЕСКИЙ СПИСОК}

[1] Грачева Н.Н., Зиновьева А.В., Полякова Е.В Актуальные вопросы льготного обеспечения граждан по государственным программам// Материалы VIII международной научнопрактической конференции «Решение проблем развития предприятий: роль научных исследований». 2016. С. 1 - 9.

[2] Ковалева К.А., Наркевич И.А., Немятых О.Д., Васягина Ю.А. Анализ федеральных программ льготного лекарственного обеспечения населения//Ремедиум. 2019. Т.3. С.54-58.

[3] Феоктистова Ю. В., Поддубный Е. А., Манеева Е. С., Елисеева Е. В. Нормативно-правовые и организационные основы льготного лекарственного обеспечения отдельных категорий граждан// Таврический медикобиологический вестник. 2018, Т. 21, № 4. С.120127

[4] Хабриев Р.У., Суходолов А.П., Спасенников Б.А., Безмельницына Л.Ю., Мешков Д.О.

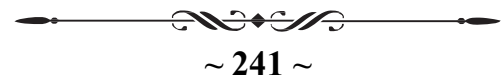


[5] Jeffrey P. Bratberg. Optimizing community pharmacist-led population health// J Am Pharm Assoc. 2019. Vol. 59 (6). P. 765-766.

[6] Strand M.A., Davidson K.M., Schulze N. Linking pharmacists to the delivery of public health services//J Am Pharm Assoc. 2017. V. 57 (6). P. 742-746.
Система принятия решений в программе льготного лекарственного обеспечения// Известия Байкальского государственного университета. 2018. Т.28, №1. С. 6-11.

[5] Jeffrey P. Bratberg. Optimizing community pharmacist-led population health// J Am Pharm Assoc. 2019. V. 59 (6). P. 765-766.

[6] Strand M.A., Davidson K.M., Schulze N. Linking pharmacists to the delivery of public health services//J Am Pharm Assoc. 2017. V. 57 (6). P. 742-746.

Author Contributions. Filina I.A. - literature review, writing a text; Ustinova L.P. - sample collection and material processing, study design; Varenyk G.V. - sample collection and material processing, writing a text. Maltsev E.A. - sample collection and material processing.

Conflict of Interest Statement. The authors declare no conflict of interest.

Ustinova L.P. - SPIN ID: 8477-9882; ORCID ID: 0000-0001-8966-0766

Research interests, number of main publications: 6 publications were published, 1 in the Higher Attestation Commission and 5 in conference proceedings.

Filina I.A. - SPIN ID: 2899 - 8564; ORCID ID: 0000-0002-4227-5059

Research interests, number of main publications: circulation of medicinal products, more than 50 publications were published, 2 of them in SCOPUS, 21 in the Higher Attestation Commission and 22 in conference proceedings.

Varenyk G.V.- SPIN ID: 2112-7313; ORCID ID: 0000-0001-6318-3949

Research interests, number of main publications: circulation of medicinal products, more than 15 publications were published, 9 in the Higher Attestation Commission and 7 in conference proceedings.

Maltsev E.A. - SPIN ID: 1846-6911; ORCID: 0000-0001-5983-5246

Research interests: circulation of medicinal products, more than 6 publications were published, 6 in the conference proceedings.

Вклад авторов. Филина И.А. - обзор литературы, написание текста; Устинова Л.П. - сбор и обработка материалов, дизайн исследования; Вареных Г.В. - сбор и обработка материалов, написание текста; Мальцев Е.А. - сбор и обработка материалов.

Заявление о конфликте интересов. Авторы заявляют об отсутствии конфликта интересов.

Устинова Л.П. - SPIN ID: 8477-9882; ORCID ID: 0000-0001-8966-0766

Сфера научных интересов, количество основных публикаций: обращение лекарственных средств, опубликовано 6 статей, из них 1 в ВАК и 5 в материалах конференций.

Филина И.А. - SPIN ID: 2899 - 8564; ORCID ID: 0000-0002-4227-5059

Сфера научных интересов, количество основных публикаций: обращение лекарственных средств, опубликовано более 50 публикаций, из них 2 в СКОПУС, 21 в ВАК и 22 в материалах конференций.

Вареных Г.В. - SPIN ID: 2112-7313; ORCID ID: 0000-0001-6318-3949

Сфера научных интересов, количество основных публикаций: обращение лекарственных средств, опубликовано более 15 статей, из них 9 в ВАК и 7 в материалах конференций.

Мальцев E.A. - SPIN ID: 1846-6911; ORCID: 0000-0001-5983-5246

Сфера научных интересов: обращение лекарственных средств, опубликовано более 6 статей, из них 6 в материалах конферениий.

For citation: Ustinova L.P., Filina I.A., Varenyk G.V., Maltsev E.A. ANALYSIS OF POPULATION SATISFACTION WITH THE QUALITY OF PROVIDING SERVICES ON PREMIUM MEDICINAL PROVISION // Medical \& pharmaceutical journal "Pulse". - 2021. - Vol. 23. - №6. - C. 240-246. doi: 10.26787/nydha-2686-6838-2021-23-6-240246.

Для цитирования: Устинова Л.П., Филина И.А., Вареных Г.В., Мальцев Е.А. АНАЛИЗ УДОВЛЕТВОРЕННОСТИ НАСЕЛЕНИЯ КАЧЕСТВОМ ПРЕДОСТАВЛЕНИЯ УСЛУГ ПО ЛЬГОТНОМУ

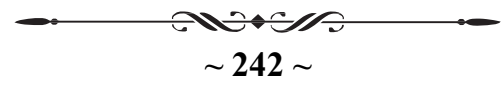


ЛЕКАРСТВЕННОМУ ОБЕСПЕЧЕНИЮ // Медико-фармацевтический журнал "Пульс". - 2021. - Т. 23. - №6. - С. 240-246. doi: 10.26787/nydha-2686-6838-2021-23-6-240-246.

Введение. Терапия лекарственными препаратами является одним из главных составляющих медицинской помощи населения, а обеспечение лекарствами служит важным показателем еe доступности и качества. Бесплатное оказание лекарственной помощи осуществляется на основании правовой базы и регулируется как на региональном, так и на федеральном уровне. Согласно статистическим данным Департамента здравоохранения Орловской области, общая численность льготных категорий граждан довольно внушительна, и составляет почти $10,0 \%$ всего населения Орловской области. Большинство из них являются инвалидами тех групп, которые имеют право на государственную помощь в соответствии с Федеральным законом от 19 июля 1999 года № 178-Ф3 «О государственной социальной помощи». Численность льготных категорий населения определяется тем количеством граждан, которые имеют право на получение набора социальных услуг и не отказались от них. При организации лекарственной помощи населению, имеющему льготное лекарственное обслуживание, необходим регулярный сбор информации о том, довольны ли льготники доступностью и ассортиментом бесплатных препаратов, качеством обслуживания в медицинских и аптечных организациях, эффективностью лечения и т.д. $[1,5,6]$.

Цель настоящего исследования - анализ удовлетворенности населения качеством предоставления услуг по льготному лекарственному обеспечению.

Материалы и методы. В качестве информационной базы взяты труды отечественных и зарубежных ученых в области льготного лекарственного обеспечения, а также нормативно-правовая и организационная документация по фармацевтической деятельности. Метод анкетирования послужил основным методом исследования, в результате которого было опрошено 119 респондентов аптечных организаций Орловской области, получающих лекарственные препараты в рамках программы льготного лекарственного обеспечения.

Результаты и обсуждение. По результатам статистической обработки данных был получен социально-демографический портрет пациентов, получающих лекарственные препараты (ЛП) бесплатно или с 50,0\% - скидкой.

Из таблицы 1 видно, что большую часть льготников составляют женщины (56,0\%) в возрасте от 40 до 64 лет $(45,0 \%)$, среди которых $40,0 \%$ работающие и проживающие в городе $(79,0 \%)$. Из всех опрошенных большая часть $(71,0 \%)$ имеют сопутствующие заболевания помимо основного, а стаж получения льготных препаратов у большинства составляет от 5 до 10 лет $(45,0 \%)$.

Таблица 1

Социально-демографическая характеристика населения, \%

\begin{tabular}{|l|l|l|l|}
\hline Показатель & Количество \% & Показатель & Количество \% \\
\hline Пол: & & Возраст (лет): & \\
Мужчины & 44,0 & $18-39$ & 29,0 \\
& 56,0 & $\begin{array}{l}\text { Женщины } \\
\text { - } 65 \text { лет }\end{array}$ & 45,0 \\
\hline Социальное положение: & & Населенный пункт проживания: & 26,0 \\
$\begin{array}{l}\text { Учащийся } \\
\text { Работающий }\end{array}$ & 12,0 & Село & \\
Безработный & 40,0 & Город & 21,0 \\
Пенсионер & 3,0 & & 79,0 \\
Инвалид & 28,0 & & \\
& 17,0 & & \\
\hline Стаж льготника (лет): & 34,0 & Сопутствующие заболевания: & \\
$<5$ лет & Да & \\
$5-10$ & 45,0 & Нет & 71,0 \\
\hline
\end{tabular}




\begin{tabular}{|l|l|l|l|}
\hline $10-20$ & 17,0 & & 29,0 \\
$>20$ лет & 4,0 & & \\
\hline
\end{tabular}

Table 1

Socio-demographic characteristics of the population, $\%$

\begin{tabular}{|l|l|l|l|}
\hline Index & $\%$ & Index & $\%$ \\
\hline Floor: & & Age (years old): & \\
Men & 44,0 & $\begin{array}{l}18-39 \\
40-64\end{array}$ & 29,0 \\
Women & 56,0 & 65 years old & 45,0 \\
& & $\begin{array}{l}\text { Locality of residence: } \\
\text { Social status: }\end{array}$ & \\
Student & 12,0 & Village & 21,0 \\
Working & 40,0 & City & 79,0 \\
Unemployed & 3,0 & & \\
Retiree & 28,0 & & \\
Disabled & 17,0 & & 71,0 \\
\hline Beneficiary's experience (years old): & & Accompanying illnesses: & \\
$<5$ лет & 34,0 & Yes & 29,0 \\
$5-10$ & 45,0 & Not & \\
$10-20$ & 17,0 & & \\
$>20$ лет years old & 4,0 & & \\
\hline
\end{tabular}

Важным показателем удовлетворенности пациентов является оценка деятельности специалистов медицинской организации (МО), где наблюдается больной. Так, почти у половины посетителей $(49 \%)$ возникали трудности при записи на прием к врачу. На вопрос о том, бывают ли случаи отказа в выписке льготного рецепта, положительно ответили $29,0 \%$ опрошенных пациентов; 45,0\% анкетируемым приходилось затрачивать продолжительное время на оформление льготного рецепта на ЛП решением BK.

Согласно Приказу Министерства здравоохранения РФ от 11 июля 2017 г. № 403н «рецепт на лекарственный препарат, входящий в минимальный ассортимент лекарственных препаратов для медицинского применения, необходимых для оказания медицинской помощи, обслуживается в течение пяти рабочих дней со дня обращения лица к субъекту розничной торговли; рецепт на лекарственный препарат, отпускаемый бесплатно или со скидкой и не вошедший в минимальный ассортимент лекарственных препаратов для медицинского применения, необходимых для оказания медицинской помощи, обслуживается в течение десяти рабочих дней со дня обращения лица к субъекту розничной торговли; рецепты на лекарственные препараты, назначаемые по решению врачебной комиссии, обслуживаются в течение пятнадцати рабочих дней со дня обращения лица к субъекту розничной торговли». Результаты анкетирования показали, что только $51,0 \%$ респондентов удовлетворены уровнем обеспечения ЛП. На вопрос о том, своевременно ли получаете ЛП по льготным рецептам, 54,0\% всех респондентов ответили отрицательно (отложено на срок более 10 дней). Такие ситуации влекут за собой несвоевременную фармакотерапию, использование скорой медицинской помощи или лечение в стационаре [3].

Для большинства пациентов удалённость расположения аптеки, обеспечивающей льготные рецепты, от дома составляет по времени до 30 минут $(41,0 \%)$ и до 1 часа $(37,0 \%)$. Можно сказать, что территориальная доступность лекарственного обеспечения для большинства жителей региона является удовлетворительной. «Доступность лекарственной помощи льготной категории граждан аптекой включает в себя своевременность внесения сведений в реестр, наличие препарата при первом обращении, организацию обслуживания, время ожидания обслуживания, полноту оказания консультационных услуг и другое $[2,4] »$. В результате анкетирования $80,0 \%$ респондентов устраивает обслуживание в аптечной организации по отпуску льготных ЛП; 81,0\% пациентов удовлетворены качеством и полнотой информации, полученной от врача, аптечного работника.

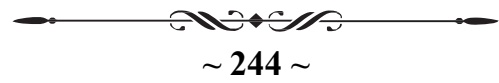


В ходе анкетирования выяснилось, что почти половина опрошенных респондентов $(48,0 \%)$ не удовлетворены лечением ЛП по

рецептам, выписанным в рамках программы льготного лекарственного обеспечения согласно причинам, которые представлены на рисунке 1.

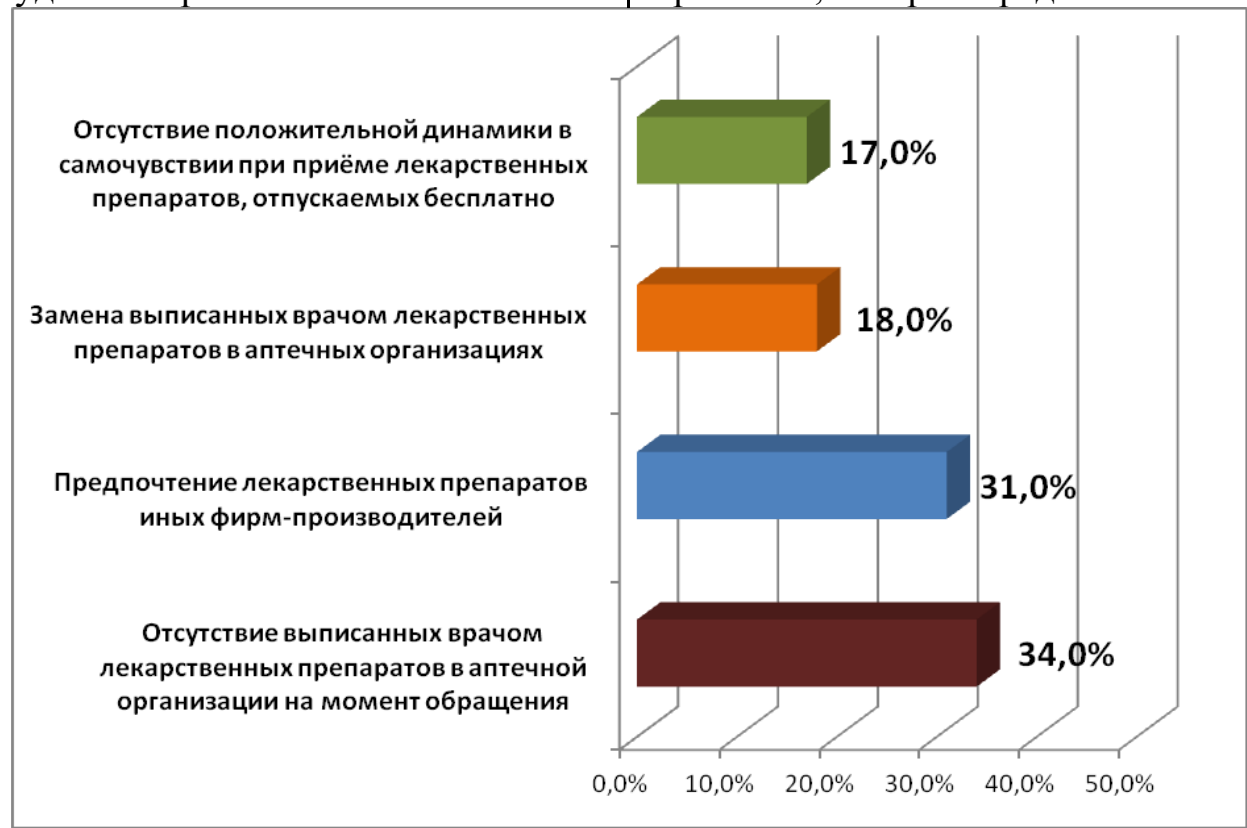

Рис.1 - Причины неудовлетворенности пациентов лечением лекарственными препаратами в рамках льготного лекарственного обеспечения

Fig. 1 - Reasons for patient dissatisfaction with drug treatment under preferential drug provision

Назначение и применение ЛП в рамках бесплатного лекарственного обеспечения осуществляется на основе стандартов медицинской помощи, при этом учитывается включение лекарственного препарата в перечень жизненно необходимых и важнейших лекарственных препаратов (ЖНВЛП). Поэтому важным критерием качества оказываемых медицинских услуг является ассортимент льготных ЛП. Согласно опросу, лишь 39,0\% анкетированных считают, что ассортимент ЛП является достаточным для лечения основного заболевания. Лекарства, не входящие в перечень ЖНВЛП, могут быть назначены решением врачебной комиссии (BК) медицинской организации в случаях их замены из-за индивидуальной непереносимости или по жизненным показаниям; (45,0\%) респондентов ответили, что им приходилось затрачивать продолжительное время на оформление рецепта на льготный ЛП решением ВК. Среди всех опрошенных 24,0\% льготников отказались от получения бесплатных ЛП на текущий год в пользу ежемесячной денежной выплаты, однако 40,0\% планируют отказаться от денежной выплаты в пользу возврата льготного лекарственного обеспечения.
Выводы. В результате полученных данных выявлено, что:

1. Имели трудности при записи на прием к врачу 49,0\% респондентов; на случаи отказа в выписке льготного рецепта указали 29,0\% опрошенных; $\quad 45,0 \% \quad$ анкетируемым приходилось затрачивать продолжительное время на оформление льготного рецепта на ЛП решением ВК.

2. Большинство респондентов $(54,0 \%)$ не получают своевременно ЛП по льготным рецептам (отложено на срок более 10 дней). Почти половина опрошенных респондентов (48,0\%) не удовлетворены лечением ЛП по рецептам, выписанным в рамках программы льготного лекарственного обеспечения, по причине отсутствия выписанных врачом ЛП в аптечной организации на момент обращения и предпочтения ими ЛП иных фирм-производителей. Большая часть анкетируемых $(61,0 \%)$ считает ассортимент льготных ЛП недостаточным для лечения своего основного заболевания.

3. Некоторых респондентов $(20,0 \%)$ не устраивает обслуживание в аптечной организации; 19,0\% недовольны качеством и полнотой информации о ЛП, полученной от врача и аптечного работника. 
4. Полученные результаты были доведены до руководства медицинских и аптечных организаций, обслуживающих льготные категории граждан в Орловской области. Авторами исследования разработана Стандартная операционная
«Порядок реализации лекарственных препаратов льготным категориям граждан», которая способствует оптимизации качества обслуживания в аптеках населения, получающего ЛП в рамках программы льготного лекарственного обеспечения.
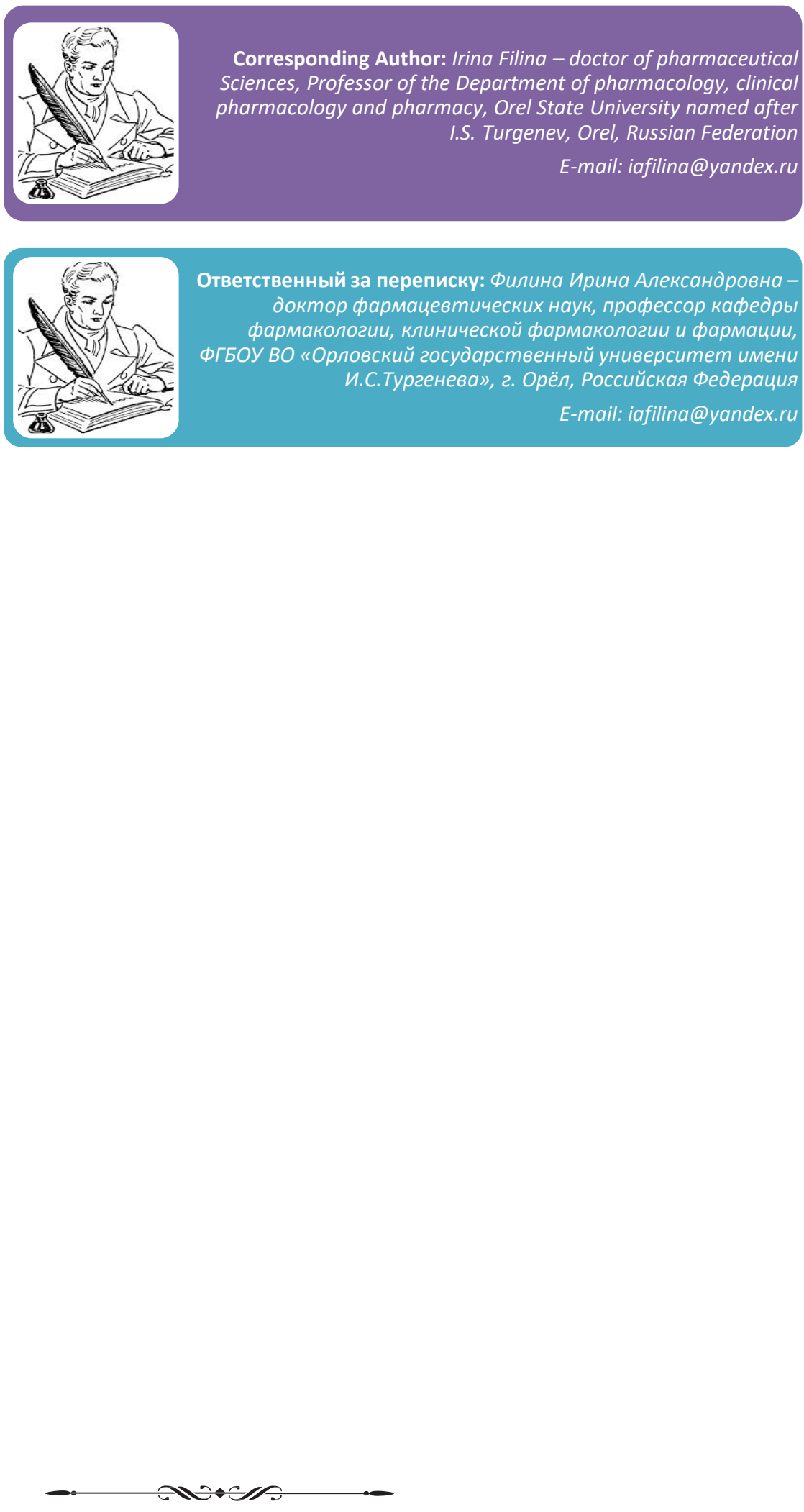